\title{
IMMUNOCHEMICAL MECHANISMS INVOLVED PENICILLIN HYPERSENSITIVITY IN EXPERIMENTAL ANIMALS AND IN HUMAN BEINGS
}

\author{
BERNARD B. LEVINE \\ Assistant Professor of Medicine, New York University School of Medicine, New York, N.Y.
}

IN addition to its importance in clinical medicine, the human penicillin allergy system provides a comparatively well defined haptenic system for the study of fundamental immune mechanisms involved in human allergic diseases. Also, the understanding of the mechanisms involved in clinical allergic reactions to penicillin would in turn allow for more rational approaches to the prediction, diagnosis, prevention and management of these diseases. Penicillin allergies are, of course, immune diseases, i.e., they follow antibody/ antigen reactions taking place in relation to tissue. Accordingly, the following kinds of information are important in elucidating their immune mechanisms: (1) the chemical mechanism of antigenicity of penicillin; (2) the antigenic specificities of immune responses to penicillin; (3) the kinds and quantities of antibodies synthesized in response to the administration of penicillin and, (4) the relationship of the nature of the immune responses to the occurrence of clinical allergic reactions. Studies in experimental animals provided a basis for later studies in human beings, and broaden the biological significance of the results obtained. This paper will deal with the chemical mechanisms of antigenicity of penicillin, the antigenic specificities of the immune responses of guinea pigs, rabbits and human beings to administration of penicillin, our early views on the possible immune mechanisms involved in clinical allergic reactions to penicillin, and the clinical implications of these views.

\section{Antigenicity of Benzyl Penicillin}

In order for low molecular weight chemicals to induce immune responses, they must first combine irreversibly with tissue macromolecules (most probably, proteins) to form the haptenprotein conjugates which induce the synthesis of specific antibodies. This view is widely accepted among immunologists, and is based on much data which show that of a large number of simple chemicals, only those which combine, through covalento linkages, with proteins or with protein- $\overrightarrow{-}$ model compounds are capable of induc- $\omega$ ing immune responses (Landsteiner, 1945: Gell, Harrington and Pitt-Rivers, 1946; Eisen 3 1959). With regard to many drugs which cani cause allergic reactions, but would appear from their structural formulæ to be incapable of reacting irreversibly with proteins, it is reason-음 able to postulate that either a trace contami- $\vec{A}$ nant, a degradation product, or an interme-o diate metabolite of the drug may be the actual protein-reactive material (Landsteiner, 1945). $\overrightarrow{-}$ With regard to the antigenicity of benzyl penicillin (penicillin G, PG), the available evidenced indicates that PG first rearranges to form

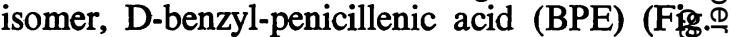
1), which is a highly reactive compound, and $\overrightarrow{0}$ which is the protein-reactive compound res ponsible for the induction of immune responses to PG (Levine, 1960a, 1960b, 1961). This rearrangement appears not to be dependent upon in vivo enzymatic catalyses. It occurs in vitro as well as in vivo, and its rate is increased by low $\mathrm{pH}$ and by the presence of caiions such as $\mathrm{Cu}^{++}, \mathrm{Zn}^{++}, \mathrm{Fe}^{++} \overrightarrow{0}$ (Florey et al., 1949; Clarke, Johnson and Robinson, 1949). BPE reacts irreversibly mainly with lysine $\varepsilon$-amino groups of proteins to form benzylpenicilloyl-amine haptenic groups (Levine, 1961; Levine and Ovary, 1961). (Fig. 1). Other haptenic groups are formed to a lesser 3 . extent, as will be taken up below. Alternatively, it may be considered that PG might⿳ react to form benzylpenicilloyl haptenic groups by a direct addition reaction of the $\beta$-lactam carbonyl to lysine amine residues of proteins. This view of the antigenicity of $P G$ appears less likely from the following lines of evidence: (1) although this latter reaction doesn proceed at $\mathrm{pH} 11.5$ with a half life in the orderN of minutes (Levine, unpublished data), ito would proceed very much slower at $\mathrm{pH} 7.5<$ probably in the order of $1 / 10,000$ th as rapidlyc as at $\mathrm{pH} 11.5$; (2) PG does rearrange to BPE under physiological conditions in vitro (Levine, 


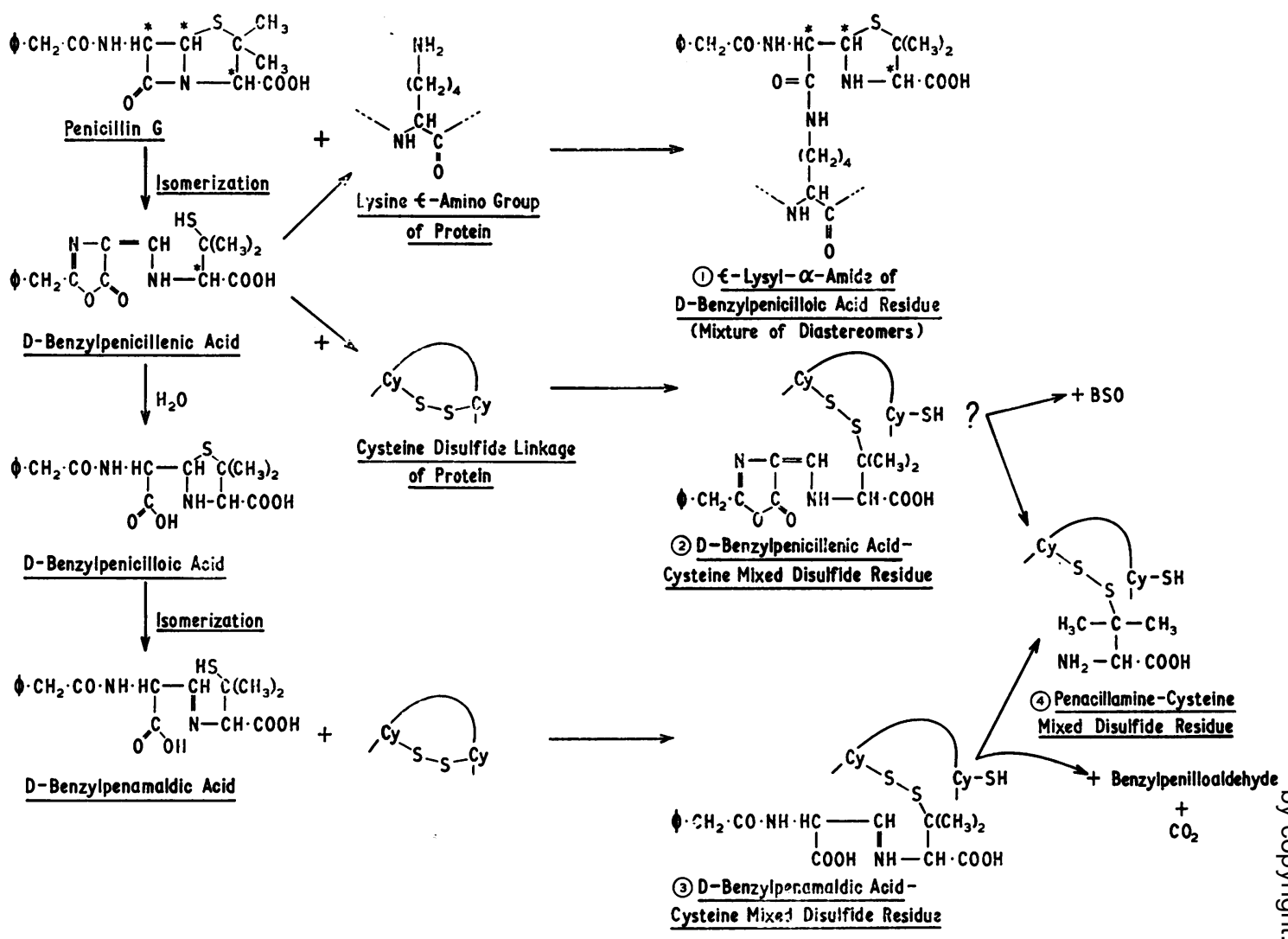

FIG. 1.-Postulated chemical pathways leading to the formation of "penicillin antigens".

1960b; Clarke et al., 1949) and undoubtedly also in vivo. The rate of rearrangement in vivo is unknown. Based on the data of Eagle (1947) and on arguments given previously (Levine, 1960a), it may be as much as $10 \%$ per hour; (3) BPE is an exceedingly chemically reactive compound. In aqueous solution, $\mathrm{pH} 7.5$ at $37^{\circ} \mathrm{C}$. it is hydrolyzed at the rate of $11 \%$ per minute (Levine, 1961) at least 550 times more rapidly than is PG under identical conditions (Benedict, Schmidt and Coghill, 1946); (4) sensitization of guinea pigs (for allergic contact dermatitis, Levine, 1960a), rabbits (for serum antibodies, Levine, 1961b; 1964a) and human beings (for skin sensitizing antibodies, Levine and Price, 1964) and probably also for serum 7S and 19S antibodies (Levine, unpublished data) induces the formation of antibodies specific for a mixture of diastereoisomers of the benzylpenicilloyl haptenic group rather than for the $\alpha$-diastereoisomer alone. If the "complete penicillin antigens" which induce antibody formation, were formed by the direct addition of the $\beta$-lactam carbonyl of penicillin to amine residues of protein, the resulting benzylpenicilloyl haptenic groups would be formed as a diastereoisomer (Levine, 1962). If the reaction proceeded through the intermediate formation of benzylpenicillenic acid, the benzylpenicilloyl groups would be formed as a diasteroisomeric mixture (Levine, 1961a). The finding that antiPG antibodies arespecific for a diastereoisomeric mixture of benzylpenicilloyl groups is thus consistent with the view that PG combines with tissue proteins through the intermediate BPE. An unequivocal proof of this view, however, requires precise measurements of the rate of rearrangement of PG to BPE under conditions which simulate physiological conditions in vitro, e.g. the reaction solution should contain traces of $\mathrm{Cu}^{++}$, etc. These arguments given above, although indicating that PG reacts with tissue proteins primarily through 
the reactive intermediate $\mathrm{BPE}$, do not exclude the possibility that $P G$ may also react directly with proteins to some extent.

\section{Antigenic Specificities of Immune Responses to PG in Experimental Animals}

Guinea pigs can be made allergic (contact dermatitis type) to PG by repeated percutaneous application of solutions of PG in a solvent containing the anionic detergent Tween 80 (Levine, 1960a).

The haptenic specificities of allergic contact dermatitis reactions were inferred from the patterns of allergic cross-reactivity among PG and its highly purified degradation products, as well as from the chemical reactivities of PG and its degradation products. The observation that groups of guinea pigs sensitized with PG or with BPE both give identical allergic reactions to patch tests with PG and with BPE (i.e., the two groups of guinea pigs are "allergically equivalent" - see Levine (1960a) for more detailed descriptions) indicates that the allergic contact reactions are specific for the diastereoisomeric benzylpenicilloyl groups. In addition, specific allergic cross reactivity among PG, sodium benzylpenicilloate and D-penicillamine is observed (Levine, 1960a). These data and the finding that benzylpenicilloate reacts with cystine (through the intermediate formation of benzylpenamaldic acid-cysteine mixed disulfide) to form D-penicillamine cysteine mixed disulfide (Levine, 1960c) (Fig. 1), indicates that the D-benzylpenamaldic acid-cysteine mixed disulfide and the D-penicillamine-cysteine mixed disulfide groups are also haptenic determinants. In addition, the benzylpenicillenic aciddisulfide group (Fig 1) is a possible fourth haptenic determinant. In the few studies done on human beings, two of three patients with allergic contact dermatitis to $\mathrm{PG}$ reacted also to patch tests with D-penicillamine (Levine and Eisen, unpublished data), which suggests that a similar haptenic heterogeneity exists in human beings with allergic contact dermatitis to penicillin. The foregoing observations indicate that the antigenic specificity of allergic contact dermatitis induced by the simple chemical compound, benzyl penicillin, is directed toward at least three different haptenic determinants (Fig. 1). This heterogeneity of haptenic antigenic specificity has been found also for the skin sensitizing antibodies induced in human beings made hypersensitive to benzyl penicillin (see below). From considerations of the chemical reactivities of PG and its degradation products with protein-model compounds (Levine, 1960a, 1960b; Florey, क 1949), it is likely that most of the penicillin that combines irreversibly with tissue proteins form benzylpenicilloyl haptenic groups, whereas $\stackrel{5}{+}$ only a comparatively small fraction of the PGO that reacts forms the penamaldic acid and 을 penicillamine mixed disulfide haptenic deter- $\frac{\bar{s}}{\sigma}$ minants. On this basis, the benzylpenicilloyl $\stackrel{\odot}{\propto}$ group has been termed the major antigenic determinant, and the two mixed disulfide determinants (including other unidentified trace $?$ haptenic determinants, see below) have been $\vec{\omega}$ termed the minor antigenic determinants of $\stackrel{\sim}{D}$ penicillin hypersensitivity Levine and Price, 1964; Siegel and Levine, 1964).

\section{Antigenic Specificity of Rabbit Anti-benzyl- penicillin Antibodies}

Rabbits can be immunized to PG by injection of PG emulsified in complete Freund's adjuvants followed by booster injections of aqueous penicillin or aqueous suspensions of procaine $\varepsilon$-penicillin. Under these conditions, sera containing as much as $400-500 \mu \mathrm{g} . / \mathrm{ml}$. of $\mathbb{\Phi}$ antibody protein can be obtained (Levine an\& Ovary, 1961). These antibodies are specifi $\mathbb{\Phi}$ for the benzylpenicilloyl haptenic group as $\vec{\varphi}$ shown by quantitative precipitin techniques (Levine and Ovary, 1961), by passive cutaneous anaphylaxis (PCA) in guinea pigs (Levine and Ovary, 1961; Levine, 1964a), and by passive hemagglutination (De Weck, 1962). Benzylpenicilloyl haptenic specificity of these immunological reactions was confirmed by quantitative hapten inhibition techniques (Levine and Ovary, 1961; Levine, 1964a; DeWeck, 1962) using benzylpenicilloyl- $\varepsilon$-aminocaproate (Levine, 1962) as the univalent hapten. The anti-benzylpenicilloyl antibodies show specificity also for the lysine side chain of proteins through which benzyl-penicilloyl groups are predominantly bound to protein (Levine and Ovary, 1961; Levine, 1962; 1963). Other studies have demonstrated that antibenzyl-penicilloyl antibodies produced by immunization of rabbits with benzylpenicilloylrabbit serum albumin conjugates are specific also for structural areas of the carrier protein (carrier specificity) (Levine, 1963). Some evidence indicates that anti-benzylpenicilloyl antibodies produced by immunization of rabbits with PG also show carrier specificity to as yet unidentified carrier proteins (Levine, 1964a). Thus, available evidence indicates that the bulk of antibodies produced by immuniza-

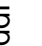


tion of rabbits with PG show immunological specificity for a rather large antigenic unit comprised of the entire benzylpenicilloyl haptenic group, the lysine side chain, and possibly also for structural configurations of as yet undefined autologous protein carriers to which benzylpenicilloyl groups are bound. No antibodies specific for the benzylpenicillenic acid mixed disulfide or the D-penicillamine mixed disulfide haptenic groups were detected in rabbit anti-PG sera by precipitation and by PCA (Levine and Ovary, 1961; Levine, 1964a), nor could indirect evidence of antibodies specific for the D-benzylpenamaldic acid-mixed disulfide haptenic group be obtained (Levine and Ovary, 1961). DeWeck (1962) stated that he has detected anti-benzylpenicillenic acid disulfide antibodies in rabbit anti-PG sera, but no supporting data was given.

\section{Antigenic Specificities of Human Anti- Penicillin Antibodies.}

On an operational basis, two different classes of anti-PG antibodies can be detected in human beings, i.e. skin sensitizing antibodies and serum antibodies detectible by passive hemagglutination. From studies in other antigenic systems, skin sensitizing antibodies are believed to be $\gamma 1 \mathrm{~A}$ globulins (Heremans and Vaerman, 1962; Fireman, Vannier and Goodman, 1963). Antigenic specificities of skin sensitizing antibodies can be studied by direct skin test for wheal-and-flare reactions, and by passive transfer of sera from patients with recent allergic reactions to the skin of nonsensitive human recipients. Three kinds of antigenic specificities of skin sensitizing antibodies have been found by these studies:

(1) Benzylpenicilloyl-specific antibodies were detected by direct skin tests (Levine and Ovary, 1961; Levine and Price, 1964; Parker, Shapiro, Kern and Eisen, 1962) and by passive transfer techniques (Siegal and Levine, 1964). Skin test reagents which can be used for detection of benzylpenicilloyl (BPO) specific antibodies are multivalent BPO-haptens such as BPO-protein conjugates and BPO-polylysine. Succinylated BPO-polylysine conjugates of poly-L-lysine poly-D-lysine appear, at present, to be the most useful clinical reagents for the detection of BPO-specific skin reactivity. They are effective elicitors of BPO-specific wheal-andflare reactions (Parker et al., 1962; Levine and Price, 1964), they are virtually non-irritating, and they are incapable of inducing immune responses in experimental animals (Levine, 1964b; Parker and Thiel, 1963), and probably also in man. BPO-specificity of these wheal-and-flare reactions (Levine and Ovary, $\mathbb{D}$ 1961; Levine and Price, 1964; Parker et al., $\subseteq$ 1962; Siegal and Levine, 1964) as well $\vec{\Rightarrow}$ as specificity for a diastereoisomeric mixture of $\stackrel{\sim}{+}$ BPO groups (Levine and Price, 1964) was confirmed by hapten inhibition experiments, using benzylpenicilloyl aminocaproate (Levine, 1962) as the univalent hapten. Other experiments indicate that the anti-BPO skin-sensitizing antibodies show specificity also for structural configurations of the autologous carrier proteins which induced the synthesis of antibody (carrier specificity) (Levine and Price, 1964). Carrier specificity toward human serum albumin was indicated in one patient (Levine and Price, 1964), towards human gamma globulin in another (Levine and Ovary, 1961), and to as yet unidentified proteins in others (Levine and Price, 1964). Since skin sensitizing antibodies may mediate some kinds of allergic reaction to penicillin, the indication that anti-BPO antibodies are specific also for structural configurations of homologous proteins would suggest that some allergic reactions to penicillin are, in part, auto-immune diseases. The exact clinical significance of carrier specificity is not yet known.

(2) Another group of patients show specific wheal-and-flare skin reactivity bs direct skin test to crystalline potassium benzyl:penicillin (KPG) and to crystalline sodium benzylpenicilloate (free from PG impurity) (Levine and Price, 1964, and unpublished data). Two of these patients (out of eight tested) gave weak reactions also to a multivalent D-penicillamine conjugate (Levine and Price, 1964, and unpublished data). Two other patients were tested and failed to react to $D$ penicillamine, penilloaldehyde and to benzyloxazolone (unpublished data). This pattern of reactivity indicates specificity to the $D$ benzylpenamaldic-cysteine mixed disulfide and the D-penicillamine-cysteine mixed disulfide groups (Levine and Price, 1964). However, this specificity must be confirmed by more direct experiments. The postulated chemical pathway leading to the formation of these haptenic groups is shown in Fig. 1.

(3) The third kind of antigenic specificity was observed by passive transfer technique (Siegal and Levine, 1964). Human skin sites passively sensitized with serum from patients with recent immediate systemic allergic reactions to penicillin, gave whealand-flare reactions to crystalline $\mathrm{KPG}$, but not to sodium benzylpenicilloate, nor to 
multivalent conjugates of the $\mathrm{BPO}$, the $\mathrm{D}$ benzylpenicillenic acid-disulfide or the Dpenicillamine haptenic groups. The antigenic specificities of these skin-sensitizing antibodies have not been determined. Some possibilities are discussed in Siegel and Levine (1964). In addition to these three antigenic specificities, Parker et al. (1962) have reported skin sensitizing antibodies specific for the benzylpenicillenic acid-mixed disulfide group, but this has not as yet been confirmed (Levine, 1964). Accordingly, skin-sensitizing antibodies synthesized by human beings in response to the administration of PG show considerable antigenic heterogeneity. Similar antigenic heterogeneity is seen in allergic contact dermatitis to penicillin (see above).

Hemagglutinating anti-penicillin antibodies are detected by testing serum dilutions with human group $\mathrm{O}$ red blood cells (RBC) which had previously reacted with PG (Ley, Harris, Brinkley, Liles and Cahan, 1958; Watson, Jonbert and Bennett, 1961; Van Arsdel, O'Rourke, Horan and Kamasaka, 1963, or with benzylpenicillenic acid (Fellner and Levine, unpublished data). Both methods of RBC sensitization result in BPO haptenic groups covalently linked to the RBC membrane, probably through amide linkages with amino residues of membranous proteins. It is not as yet known whether other of the possible penicillis haptenic groups are also coupled to RBC by these methods of sensitization. Hemagglutinating human anti-PG antibodies are both $19 S$ and $7 S \gamma$-globulins, with 19S antibodies occurring considerably more frequently (Heremans and Vaerman, 1962; Schwartz and Vaughan, 1963; Fudenberg and German, 1960). Anti-BPO antibodies of the $\gamma 1 \mathrm{~A}$ class may also give passive hemagglutination, but this has not yet been demonstrated. The haptenic specificity of hemagglutination anti-PG antibodies is predominantly towards the BPO group (Theil and Parker, 1962; Fellner and Levine, unpublished data). Other haptenic specificities, although likely, have not as yet been demonstrated.

\section{Immune Mechanisms of Allergic Reactions to Penicillin}

Data pertinent to this problem can be obtained from prospective and retrospective correlations of the immune status of patients with the occurrence of clinical allergic reactions. In these studies, it is important to consider separately the different types of aller- gic reactions, e.g., immediate systemic, later urticarial, serum-sickness-like, etc., since each $\mathbb{\Phi}$ kind of reaction may have distinctly different $c$. immune mechanisms. Also, it should be considered that non-immune mechanisms (i.e., vas- $\stackrel{\oplus}{\stackrel{D}{P}}$ cular sensitivity to histamine, or the activity of? the reticuloendothelial system in clearing the plasma of antigen-antibody complexes) may play a role in determining whether a patient in $\stackrel{\mathbb{2}}{2}$ whom is present the immunological factors necessary to develop an allergic reaction, does indeed develop an allergic reaction.

It appears probable, at the present time, $\vec{\omega}$ that immediate systemic allergic reactions to $\frac{\text { o }}{8}$ penicillin are mediated by skin-sensitizing ( $\gamma 1 \mathrm{~A})$ anti-PG antibodies. This statement is based on the following evidence: Siegel has $\stackrel{0}{\circ}$ reported that all of 18 patients with recent immediate systemic reactions to penicillin contained in their sera, skin-sensitizing antiPG antibodies demonstrable by passive $+\vec{r}$ transfer (Siegal, 1962). In addition, six을 other patients with this clinical history all showed direct wheal-and-flare skin 0 reactivity to penicillin skin-test reagents (Fell- $\stackrel{\mathbb{D}}{\circ}$ ner and Levine, unpublished data). This close $\frac{\mathbb{}}{3}$ association of anaphylaxis and the presence of skin-sensitizing antibodies has been found other antigenic systems in human beings (Connell, Sherman and Myers, 1962), and other mammalian species (Ovary, Benacerrafo and Block, 1963; Nussenzweig, Merryman and Bennacerraf, 1964). Of interest are the findings that the antigenic specificities of skin sensitizing anti-PG antibodies from patients with $\stackrel{\varnothing}{\varnothing}$ immediate systemic allergic reactions were $\vec{\overrightarrow{ }}$ mainly to the minor antigenic determinants 3 (i.e. skin reactions were evoked by skin test $\supset$ with KPG alone, or by KPG and sodium? benzylpenicilloate, see above) and only comparatively rarely to the benzylpenicilloyl haptenic group (Levine and Price, 1964; Siegal and Levine, 1964; Siegal, 1962). The presence of BPO-specific skin sensitizing antibodies does $\varrho$ not necessarily mean that a patient will develop

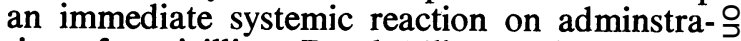
tion of penicillin. Rytel, Klion, Arlander and $>$ Miller (1963) reported that none of 26 patients with strongly positive wheal-and-flare reactions $\tilde{O}^{-}$ to penicilloyl-polylysine, and who were imme-diately thereafter injected with 1.2 million 0 units of benzathine penicillin developed imme- $\tilde{\omega}$ diate systemic reactions, although two of the 26 developed late urticarial reactions. It ise possible that these patients were protected from immediate systemic reactions by the ? presence of circulating "blocking" antibodies 
(7S and/or 19S) of the same haptenic specificity. No prospective studies are yet available correlating the presence of skin-sensitizing antibodies of minor antigenic determinant specificity to the occurrence of immediate systemic allergic reactions.

The immune mechanisms involved in late urticarial reactions, in serum-sickness-like and in other types of late allergic reactions have not as yet been elucidated. Since only $50-80 \%$ of such patients have detectable skin sensitizing antibodies (Levine and Price, 1964), immune mechanisms other than, or in addition to those involving the participation of skinsensitizing antibodies may be involved.

\section{Clinical Implications}

An important clinical application of these studies is to detect those patients who will have an allergic reaction upon the administration of penicillin. From the considerations discussed above, patients who are liable to develop immediate systemic allergic reactions should show wheal-and-flare reactions on direct skin testing with the proper antigenic reagents, providing that the patients are physiologically capable of manifesting such skin reactions. The available data on the antigenic specificities of skin-sensitizing antibodies found in patients with recent immediate systemic allergic reaction to penicillin indicates that crystalline KPG may be a more effective detector of the potential immediate reactor than is penicilloyl-polylysine (see above, and Levine and Price, 1964; Siegel and Levine, 1964). However, in order to detect the potential immediate systemic reactor, patients should be tested with both these reagents. A positive wheal-and-flare reaction does not necessarily mean that a patient will have an immediate system reaction on administration of penicillin, but it increases the probability of its occurrence. A negative skin reaction to both test materials appears, at present, to indicate that the patient will be able to receive penicillin without the occurrence of an immediate systemic reaction. However, this view is based on theoretical considerations and on experience with a relatively small number of patients. It is still possible that other skin test materials will have to be added to those now available in order to detect very rare cases of penicillin allergy. Before negative skin tests to the materials now at hand can be accepted as absolutely ruling out the possibility of an immediate systemic allergic reaction, many more patients must be evaluated. At the present time, it is not recommended that prior skin testing of patients about to be treated with penicillin be instituted as a routine clinical procedure. Before this can be done additional information must be obtained on the sensitizing capabilities of the skin test reagents. It is to be noted that negative skin tests to the reagents will not rule out the subsequent occurrence of a late urticarial or serum sickness-like allergic reaction following the administration of penicillin. At present there is no definite way to detect the patient who is more liable to suffer allergic reactions of these types.

\section{Summary}

Some current views of the immunochemical mechanisms of penicillin hypersensitivity are presented. The antigenicity of benzylpenicillin appears to depend in large part upon its ability to rearrange in vivo to its highly reactive isomer D-benzylpenicillenic acid. The postulated chemical pathways leading to the formation of the complete "penicillin antigens," and the identities of the haptenic determinants are presented. The administration of the simple chemical compound, benzylpenicillin, to experimental animals and to man leads to the production of antibodies of at least three different haptenic specificities. Some early views are given on the immune response of human beings to penicillin, on the possible immune mechanisms involved in allergic reactions to penicillin, and on the present clinical applications of these studies.

FIG. 1 is reproduced from J. exp. Med. (1960), 112, 113 by kind permission of the Editor.

\section{REFERENCES}

Benedict, R. G., SCHMidt, W. H., and COGHILl, R. D. (1946): J. Bact., 51, 291.

Clarke, H. T., Johnson, J. R., and Robinson, R., editors. The Chemistry of Penicillin. Princeton University Press, 1949.

ConNell, J. T., Sherman, W. B., and Myers, P. A. (1962): J. Allergy, 33, 365.

DeWeCK, A. L. (1962): Int. Arch. Allergy, 21, 23.

EAGLE, H. (1947): J. exp. Med., 85, 141.

EISEN, H. N., in Cellular and Humoral Aspects of the Hypersensitive States, (H. S. Lawrence, Edit.). P. B. Hoeber, Inc., New York, New York, 1959.

Fireman, P., VanNier, W. E., and Goodman, H. P. (1963): J. exp. Med., 117, 603.

Florey, H. W., Chain, E., Heatley, N. G., Jennings, W. A., SAnders, H. G., Abraham, E. P., and Florey, M. E., Antibiotics, London Oxford University Press, 1949, Vol. 2.

FudenBerg, H. H., and German, J. L. (1960) : Blood, 15,683 . 
Gell, P. G. H., Harington, C. R., and Pitt-Rivers, R. (1946): Brit. J. exp. Path., 27, 267.

Heremans, J. F., and Vaerman, J. P. (1962): Nature (Lond.), 193, 1091.

LANDSTEINER, K., The Specificity of Serological Reactions, Harvard Univ. Press, Cambridge, Mass., Rev. Edit., 1945.

LEVINE, B. B. (1960a): J. exp. Med., 112, 113.

LEVINE, B. B. (1960b): Nature (Lond.), 187, 939.

LEVINE, B. B. (1960c): Nature (Lond.), 187, 940.

LeVINE, B. B., and OVARY, Z. (1961): J. exp. Med., 114, 875.

LeVINE, B. B. (1961a): Arch. Biochem. Biophys., 93, 50

Levine, B. B. (1962): J. Med. Pharm. Chem., 5, 1025.

Levine, B. B. (1963): J. exp. Med., 117, 161 .

LEVINE, B. B., and Price, V. H. (1964): Immunology, 7,542 .

LEVINE, B. B. (1964a): Immunology, 7,527.

LEVINe, B. B. (1964b): Nature (Lond.), 202, 1008.

LeY, A., Harris, M., BRINKLeY, B., Liles, J., and CaHaN, A. (1958): Science, 127, 1118.

NussenzWeig, R. S., Merryman, C., and Benacerraf, B. (1964): J. exp. Med., in Press.
OVARY, Z., Benacerraf, B., and Bloch, K. J. (1963) J. exp. Med., 117, 951 .

PARKER, C. W., ShAPIRO, J., KeRN, M., and EISEN H. N. (1962): J. exp. Med., 115, 821 .

Parker, C. W., and ThIEL, J. A. (1963): J. lab. Clin Med. (Abstr.), 62, 988.

Rytel, M. W., Klion, F. M., Arlander, T. R., ande Miller, L. F. (1963): J. Amer. med. Ass., 186, 894.

SCHWARTZ, R. H., and VAughan, J. H. (1963): J. Amer. med. Ass., 186, 1151.

Siegel, B. B. (1962): in Allergology, Pergamon Press, ஸึ New York, 1962 , p. 125 .

Siegel, B. B., and Levine, B. B. (1964): J. Allergy; in Press.

Thiel, J. A., and Parker, C. W. (1963): Presentedo at 19th Annual Meeting, American Academy of Allergy, March 12, 1963.

Van Arsdal, P. P., O'Rourke, T. K., Horan, J. D., and Kamasaka, Y. (1963): J. Amer. med Ass.,O 186, 118 .

WATSON, K. C., Joubert, S. M., and BenNetT M. A. E. (1961): Immunology, 4, 193.

\title{
CUTANEOUS REACTIONS TO PENICILLIN
}

\author{
C. D. Calnan
}

Professor of Dermatology, University of London, The Institute of Dermatology, St. Johns Hospital for Diseases of the Skin, Leicester Square, London, W.C.2.

THE purely cutaneous reactions to penicillin are nowadays infrequent and relatively unimportant. They consist of varying degrees of acute or chronic non-specific inflammation, with or without abscess formation. They were more commonly encountered with less pure penicillins and depôt preparations sometimes injected intradermally or subcutaneously rather than intramuscularly.

All other reactions with manifestations in the skin are part of a general hypersensitivity state, although they may not necessarily be mediated by a specific antibody directed against the drug. In this regard penicillin is similar to sulphonamides in the very diverse types of adverse reaction which it is capable of producing. The factors determining the type of hypersensitivity reaction in an individual patient are mostly obscure, with the possible exception of contact dermatitis. In the latter case, the route of administration-application to the cutaneous or a muco-orificial surface-is of paramount importance.

In the United States, there are well over a hundred preparations of penicillin available too be fed, injected, spread, insufflated and sprayed $\bar{\varnothing}$ into every conceivable cavity and surface of the body (Welch, Lewis, Weinstein and Boeckman, 1957). Individuals who have neverreceived penicillin are a fast diminishing group, a result of mass production and the availability of this cheapest of all antibiotics.

\section{Incidence}

If one accepts any undesired or unexpected sequela as a reaction to the drug, the incidence $₹$ of penicillin reactions can be very high, being윽 dependent partly on the criteria of the physi- $\rightarrow$ cian. Moore's (1946) figure is $0.56 \%$, while Cormia, Jacobsen and Smith (1946) registered it as high as $60 \%$. The variation is, of course, partly due to uncritical acceptance of any $N$ symptoms and signs, and partly due to the absence of satisfactory methods of proof 0 Well controlled drug trials have shown thato

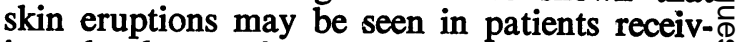
ing placebos as frequently as an active agent. Saline injections can induce the rash, arthralgia, 